\title{
Surface magnetometry with photoemission dichroism: Ultrathin epitaxial Fe-Co bcc alloys on $\mathrm{Fe}(100)$
}

\author{
Marco Liberati \\ INFM Dipartimento di Fisica dell'Università di Modena, I-4100 Modena, Italy
}

Giancarlo Panaccione and Fausto Sirotti

Laboratoire pour l'Utilisation du Rayonnement Electromagnetique, CNRS-CEA-MESR, F-91405 Orsay, France

Pilar Prieto

Dpto Fisica Aplicada, C-XII, Fac. de Ciencias, Univ. Autonoma de Madrid, E-28049 Cantoblanco, Spain

Giorgio Rossi

INFM Dipartimento di Fisica dell'Università di Modena, I-4100 Modena, Italy and Laboratorium für Festkörperphysik, ETH-Zürich, CH-8093 Zürich, Switzerland

(Received 2 July 1998; revised manuscript received 24 September 1998)

\begin{abstract}
The merits of magnetic dichroism in photoemission as a surface magnetometer are evaluated by performing a systematic investigation of the linear magnetic dichroism in the angular distribution (LMDAD) effect on the $\mathrm{Fe} 3 p$ and Co $3 p$ photoemission signal from ultrathin bcc Fe-Co alloys epitaxially grown onto $\mathrm{Fe}(100)$. [S0163-1829(99)02605-3]
\end{abstract}

\section{INTRODUCTION}

The magneto-optical effects involving core levels of ferromagnets are widely exploited in x-ray absorption [magnetic circular dichroism (MCD) ${ }^{1}{ }^{1} \mathrm{x}$-ray reflectivity, ${ }^{2,3}$ and photoemission experiments. ${ }^{4-8}$ The large spin orbit splitting and the lack of energy dispersion of the core levels make it possible to interpret the spectroscopic asymmetry obtained from magnetic dichroism experiments as a local measure of magnetization. Magneto-optical sum rules apply to the case of x-ray absorption which allows us to derive the values of $\mu_{\text {spin }}$ and $\mu_{\text {orbital }}$ from MCD experiments. ${ }^{9}$ X-ray spectroscopies do not allow us to separate surface from bulk contributions from samples of homogeneous composition. The search for a surface magnetometer leads us, as a possible choice, to consider the photoemission experiment and the magneto-optical effect in photoemission as a candidate technique due to the intrinsic surface sensitivity connected to the short mean free path of photoexcited electrons in solids and due to the high efficiency of the photoemission process when excited with soft $\mathrm{x}$ rays. ${ }^{10}$ Theoretical sum rules lack for the photoemission magnetic dichroism experiments. Among the proven magnetic dichroism effects in photoemission the one based on chiral experiments using linearly polarized radiation has practical advantages which have been previously discussed. ${ }^{5,11}$ The interpretation of the LMDAD effect on $3 p$ and $2 p$ core levels of the ferromagnetic transition metals has been based on atomic photoionization theory following original but fundamentally equivalent treatments by Cherepkov, ${ }^{12}$ Venus, ${ }^{13}$ Tamura and co-workers, ${ }^{14}$ and van der Laan. ${ }^{15}$ It has been shown that Fe $3 p$ LMDAD experiments from iron surfaces do allow us to measure the surface Curie temperature, the spin-wave stiffness (relative to the bulk) and the existence of magnetochemical shifts. ${ }^{10}$ In order to evaluate to what extent the observation of the magnetic dichroism in photoemission can be usefully exploited as an atom-selective surface magnetometer, i.e., as a diagnostic of the relative variations of magnetic moment of the atoms present at the surface, it is necessary to perform experiments on a surface system whose magnetic behavior is known, or can be closely traced with respect to a known bulk behavior.

We present here the results of a systematic study of the LMDAD of Fe $3 p$ and Co $3 p$ core-level photoemission from ultrathin epitaxial $\mathrm{Fe}-\mathrm{Co}$ alloys epitaxially grown onto $\mathrm{Fe}(100)$. Both $\mathrm{Fe}$ and Co present a large LMDAD asymmetry on the shallow $3 p$ core levels, with similar line shape as shown in previous experiments. Ultrathin bcc Fe-Co alloy films can be stabilized by epitaxy on $\mathrm{Fe}(100)$ over a wide composition range. ${ }^{16-20}$ Bulk Fe-Co alloys are characterized by a systematic variation of the total magnetic moment as well as of the local magnetic moments as a function of composition: both measurements of the saturation magnetization by Weiss and Forrer $^{21}$ and neutron-scattering data by $\mathrm{Bardos}^{22}$ for the Fe-Co alloys show a nonlinear dependence of the magnetization as a function of the increasing Co concentration with a maximum total magnetic moment of $2.4 \mu_{B}$ measured for compositions nearing $\mathrm{Fe}_{0.7} \mathrm{Co}_{0.3}$. Neutrondiffraction measurements, both with polarized and unpolarized techniques by Collins and Forsyth ${ }^{23}$ show that the nonlinear behavior of the magnetization is due principally to the increase of the magnetic moment of Fe from $2.2 \mu_{B}$ for pure Fe to $3 \mu_{B}$ for Co concentration exceeding $50 \%$, while the magnetic moment on the cobalt atom is weakly dependent upon the alloy composition. An MCD experiment by Pizzini et $a .^{24}$ confirmed these results. Several theoretical analyses of the Fe-Co alloy system have been performed. ${ }^{25-30}$ Söderlind et $a .^{25}$ calculated from first principle, using the linear muffin-tin orbital method and the virtual-crystal approximation, the spin and orbital moments of Fe-Co alloys. The enhancement of the Fe magnetic moment as it mixes with co- 
balt is due to the progressive filling of the majority spin $d$ band (pure $\mathrm{Fe}$ is a weak ferromagnet). Fe reaches the maximum magnetic moment of $3 \mu_{B}$ for $50 \%$ concentrations and does not change for further dilution in cobalt. Co has a filled majority spin $d$ band (pure Co is a strong ferromagnet): its magnetic moment changes little, i.e., from $1.65 \mu_{B}$ to $1.83 \mu_{B}$, as a function of concentration. The calculated charge transfer for the alloys is negligible.

The surface of the Fe-Co alloys has been chosen as the test system for the LMDAD method of magnetometry after having verified experimentally that the surface composition is not modified (e.g., by selective segregation) with respect to the bulk, at room temperature.

\section{LMDAD}

Several recent experimental and theoretical papers have been published on the LMDAD effect and on some applications. ${ }^{5,6,11,15,31-35}$ The LMDAD signal is obtained from chiral experiments from which one forms an asymmetry defined as

$$
A_{L M D A D}=\frac{I_{u p}-I_{\text {down }}}{I_{\text {up }}+I_{\text {down }}},
$$

where $I_{u p(\text { down })}$ are the photoemission intensities integrated over the energy range of the core level spectrum as measured with the magnetization in the upward (up) or downward (down) directions perpendicular to the plane containing both the photoelectron $k$-vector (selected by the analyzer) and the linear polarization vector of the soft $\mathrm{x}$ rays. The asymmetry defines a new order parameter that we may call the LMDAD order parameter. Its value depends on $\left\langle M_{\text {surf }}\right\rangle$, i.e., on both magnetic moment and temperature, but in a local sense due to the localized nature of the core levels and it is obviously atom specific. Its sign, when referred to the $A_{L M D A D}$ from a core level of same symmetry measured with similar kinetic energies from a ferromagnetic substrate, depends on the sign of coupling. $., 36,37$

It has been shown that the value of $A_{L M D A D}$ is fairly independent on the energy spread of the core level spectrum for moderate variations of the last. ${ }^{32}$ The energy width of the LMDAD spectrum is connected to the energy spread of the core hole multiplet which reflects the strength of the spindependent core-valence interactions, e.g., the local value of the spin magnetic moment, and is obviously independent on temperature in the ferromagnetic phase. ${ }^{10}$ The atomic model description of $3 p$ and $2 p_{3 / 2}$ core level spectra of iron and cobalt attributes the LMDAD width directly to the splitting of the $m_{j}= \pm \frac{3}{2}$ core hole sublevels, as empirically demonstrated by direct comparison of the experimental line shapes. ${ }^{11,33}$ We can define the experimental width of the LMDAD spectrum $\left(W_{L M D A D}\right)$ as the energy interval between the positive and negative peaks. The halfway energy is accordingly defined as the center of the $J=3 / 2$ multiplet. The hypothesis for using the measure of $W_{L M D A D}$ as a local measure of the spin magnetic moment is that its dependence on the actual value of the spin magnetic moment be linear within a useful range of variations of $\pm 30 \%$. $^{36}$

Photoelectron diffraction effects have been shown to modify the measured $A_{L M D A D},{ }^{38-40}$ but do not modify the energy spread of the dichroism signal. $W_{L M D A D}$ is an energy value, not an amplitude, and therefore it is the best suited measurable quantity in a LMDAD experiment to be related with the value of the local spin magnetic moment.

\section{EXPERIMENT}

The SU7 beam line at the SuperACO storage ring in LURE (Orsay) provided linearly polarized synchrotron radiation of $170 \mathrm{eV}\left(E / \Delta E \simeq 10^{3}\right)$ impinging on the sample with an angle of $50^{\circ}$ with respect to the surface normal which coincided with the direction of electron analysis. The chosen photon energy value corresponds to a large value of the photoionization differential cross section for $\mathrm{Fe} 3 p$ and Co $3 p$ and to a short mean free path (5-6 $\AA$ ) of the photoelectrons, optimizing therefore the surface sensitivity.

A (100)-oriented Fe 3\% Si single crystal was mounted to close the gap of a soft iron yoke ${ }^{6,41}$ forming a close magnetic circuit that could be excited by current pulses in a coil wound around the horseshoe. Pulses of $200 \mathrm{~mA}$ were used to saturate the sample parallel or antiparallel to the vertical $z$ axis therefore producing opposite chiralities with the fixed vectors in the $X Y$ plane ( $k$ vector and light polarization). The substrate was prepared by $\mathrm{Ar}^{+}$-ion sputtering and annealing cycles, with standard diagnostics of purity and order. The growth of the alloys was obtained in situ by coevaporation of $\mathrm{Fe}$ and $\mathrm{Co}$ from e-beam sources in a vacuum of $\sim 1$ $\times 10^{-9}$ mbar. The thickness of the alloy films was $\sim 10 \mathrm{ML}$, i.e., $15 \AA$ A. Structural tests of the evaporated surfaces were performed for homoepitaxial $\mathrm{Fe}$ on $\mathrm{Fe}(100)$ and for $\mathrm{Co} /$ $\mathrm{Fe}(100)$ as deposited in identical UHV and kinetics conditions as for the growth of the alloys, confirming the good epitaxial quality for thicknesses of $15 \AA$. The bcc structure was previously found to be the most stable for alloy thin films up to very high concentrations of Co. ${ }^{16,17,42}$ The Fe and Co sources were calibrated by a quartz crystal oscillator in the position of the target sample. The evaporation rates were stabilized to the values corresponding to the wanted alloy stoichiometry, assuming identical sticking coefficients. The sample was exposed to both $\mathrm{Fe}$ and $\mathrm{Co}$ fluxes simultaneously. A posteriori the surface composition was checked by measuring the cobalt and iron $3 p$ photoemission intensities. The photoemission calibration a posteriori agreed systematically within 5\% error with the a priori calibration of the deposit. Several growth cycles were performed for each stoichiometry to evaluate reproducibility. All the samples were grown at room temperature. The photoemission spectra were measured with the sample either at room temperature or at $150 \mathrm{~K}$.

\section{RESULTS}

In Fig. 1 we present the $\mathrm{Co}$ and $\mathrm{Fe} 3 p$ photoemission spectra for three alloys and for the reference pure metals. The Co sample is a thin film of $15 \AA$ deposited on iron. The core level spectrum shows a residual $\mathrm{Fe} 3 p$ intensity due to the substrate. The signals of iron and cobalt $3 p$ appear at kinetic energies of about 112 and $106 \mathrm{eV}$, respectively, and are considered as equivalent from the point of view of depth sensitivity.

Figures 2 and 3 display the dichroism spectra for $\mathrm{Fe}$ and 


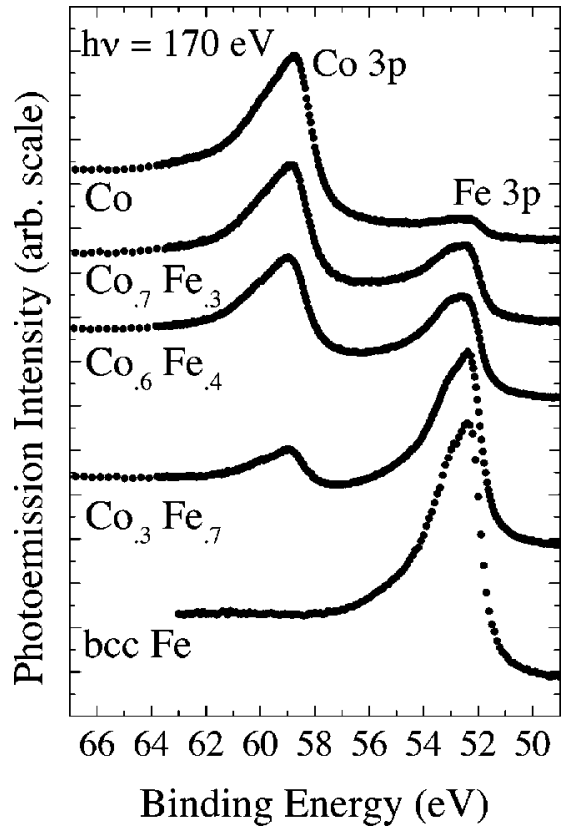

FIG. 1. Fe and Co $3 p$ core levels measured with $h \nu=170 \mathrm{eV}$ photon energy for the samples indicated in the figure.

Co, respectively. The LMDAD spectra have been aligned with respect of the negative peak which is the strongest feature to which corresponds the origin of the quoted energies in Figs. 2 and 3. The clean $\mathrm{Fe}(100)$ surface, and the thick Co layer $3 p$ LMDAD signals are taken as reference standards. The sign of $A_{L M D A D}$ for both iron and cobalt in the alloys is the same as that of the clean $\mathrm{Fe}(100)$ substrate. This confirms that alloy $\mathrm{Fe}-\mathrm{Co} / \mathrm{Fe}(100)$ interface is ferromagnetically

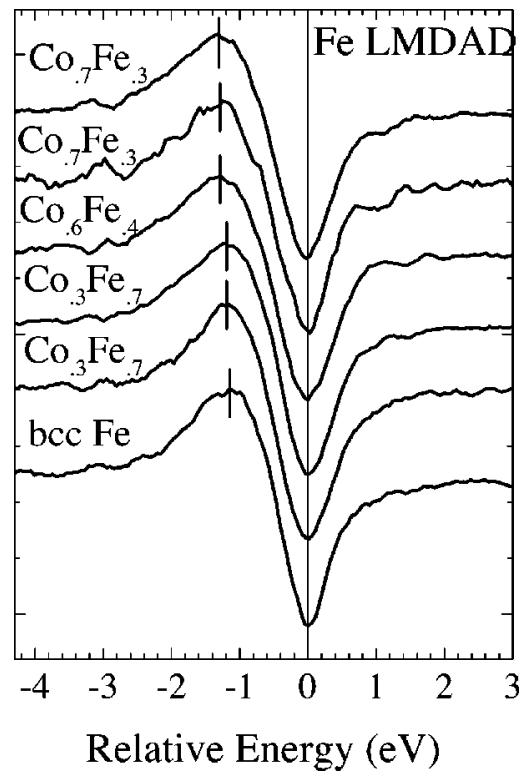

FIG. 2. Fe $3 p$ LMDAD signals normalized to the peak to peak intensity of the iron clean surface. The spectra have been aligned to the negative peak in order to show the variation of the energy splitting in the dichroic signal (vertical bars in the figure). The spectra are smoothed with an averaging on five points. The maximum asymmetry measured for $\mathrm{Fe}$ goes from $8.9 \%$ for pure iron to $13.5 \%$ for the $\mathrm{Fe}_{0.3} \mathrm{Co}_{0.7}$ alloy, showing an increasing behavior as the Co concentration increases.

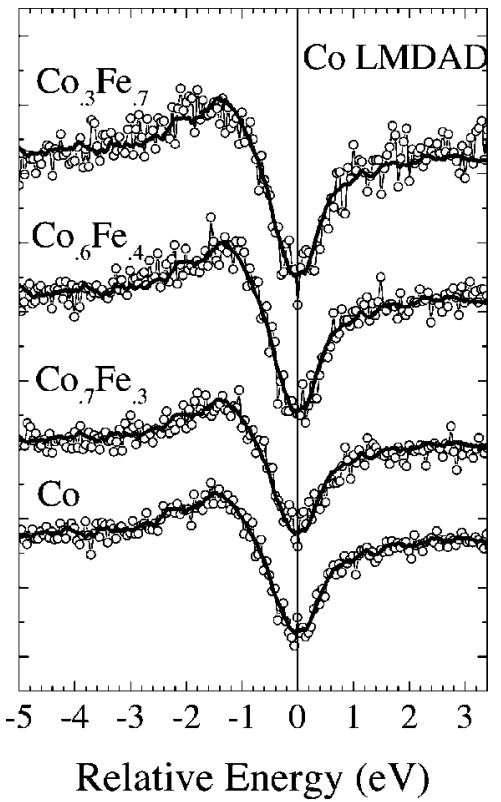

FIG. 3. Co $3 p$ LMDAD spectra (points) along with the best fit obtained by broadening the reference Co spectrum (in the bottom) applying a multiplicative factor to the energy scale of the smoothed curve. The broadening factors are taken as a measure of the $W_{L M D A D}$ variations. The measured maximum asymmetry of Co goes from $7.4 \%$ for pure cobalt to $9.4 \%$ for the $\mathrm{Fe}_{0.7} \mathrm{Co}_{0.3}$ alloy, showing an increasing behavior as the Co concentration decreases.

coupled. ${ }^{6,31}$ The general shape of the LMDAD spectrum for the Fe $3 p$ does not change for different alloys, but one can recognize a progressive widening, i.e., an increase of the distance between the negative peak and the positive one, as a function of dilution. The increase of $W_{L M D A D}$ reflects the increase in the Fe spin magnetic moment. The LMDAD spectrum for Co $3 p$ is broader with respect to $\mathrm{Fe} 3 p$ due to the $40 \%$ larger spin-orbit splitting [1.43 eV (Ref. 43)]. The analysis of the $W_{L M D A D}$ has been performed by minimizing the difference between the LMDAD spectra and the pure cobalt LMDAD spectrum which could be artificially broadened by applying a multiplicative factor to the energy scale and a rigid shift to the conventional zero of the scale (the negative LMDAD peak), i.e., by a "constant shape" approximation to the different LMDAD curves. ${ }^{36}$ The optimized multiplicative factors obtained represent the $W_{L M D A D}$ changes, i.e., the relative changes of magnetic moment between alloy and pure metal. The procedure is shown for Co in Fig. 3.

\section{DISCUSSION}

In Table I we summarize the $A_{L M D A D}$ and $W_{L M D A D}$ measured for the three Fe-Co surface alloy compositions. The value of the splitting of the LMDAD, or $W_{L M D A D}$, as a function of the Co concentration in the Fe-Co alloy, can be discussed with reference to the magnetic moments of bulk alloys.

The main reference experimental data are the results of neutron scattering by Collins and Forsyth: $:^{23}$ the Fe magnetic moment increases from $2.2 \mu_{B}$ up to $3 \pm 0.15 \mu_{B}$, i.e., it has been raised by $36 \%$ as the Co concentration increases. The saturation value of $\mu_{\mathrm{Fe}}$ is reached for stoichiometry near the 
TABLE I. Splitting and maximum asymmetry of the measured LMDAD signals of iron and cobalt.

\begin{tabular}{lcccc}
\hline \hline & \multicolumn{2}{c}{ Iron } & \multicolumn{2}{c}{ Cobalt } \\
& $\begin{array}{c}W_{L M D A D} \\
(\mathrm{eV})\end{array}$ & $\begin{array}{c}A_{L M D A D} \\
(\%)\end{array}$ & $\begin{array}{c}W_{L M D A D} \\
(\mathrm{eV})\end{array}$ & $\begin{array}{c}A_{L M D A D} \\
(\%)\end{array}$ \\
\hline $\mathrm{Fe}$ & 1.13 & 8.9 & & \\
$\mathrm{Fe}_{0.7} \mathrm{Co}_{0.3}$ & 1.19 & 8.5 & 1.39 & 9.4 \\
$\mathrm{Fe}_{0.4} \mathrm{Co}_{0.6}$ & 1.29 & 13 & 1.38 & 7.5 \\
$\mathrm{Fe}_{0.3} \mathrm{Co}_{0.7}$ & 1.29 & 13.5 & 1.40 & 7.4 \\
$\mathrm{Co}$ & & & 1.48 & 7.4 \\
\hline \hline
\end{tabular}

Fe-Co alloy. The Co magnetic moment value $\mu_{\mathrm{Co}}$ is estimated to be of $1.85 \pm 0.15 \mu_{B}$ for the Fe-Co alloy, and it shows a weak decreasing behavior for increasing $\mathrm{Fe}$ concentrations between $\mathrm{Fe}_{0.3} \mathrm{Co}_{0.7}$ and $\mathrm{Fe}_{0.7} \mathrm{Co}_{0.3}$.

Concerning our data, the following information can be deduced from Table I: the Fe total increase of $W_{L M D A D}$ as a function of alloying with $\mathrm{Co}$ is $\sim 15 \%$. The maximum value of $W_{L M D A D}$ is reached for the sample $\mathrm{Fe}_{0.4} \mathrm{Co}_{0.6}$ and it remains then constant for higher Fe dilution. The $W_{L M D A D}$ of Co $3 p$ spectrum decreases for increasing Fe concentration: it is reduced by $\sim 8 \%$ for $\mathrm{Fe}_{0.4} \mathrm{Co}_{0.6}$. For $\mathrm{Co}$ concentration lower than $60 \%$ the $W_{L M D A D}$ for Co is almost constant.

Other experiments exploiting magnetic dichroism in X-ray absorption [x-ray magnetic circular dichroism (XMCD)] have been performed on Co-Fe alloys and the dichroism has been related to magnetization. Plots of normalized XMCD on both $\mathrm{Fe}$ and $\mathrm{Co} K$ edges $^{24}$ as well as on $L_{2,3}$ edges ${ }^{44}$ versus alloy composition give the same qualitative trends as measured by neutrons but were not analyzed quantitatively in terms of moments. The large variations of XMCD signal are related to the changes of the orbital magnetic moment which can have larger relative variations than the spin moment in ultrathin films or at the surface of transition metal ferromagnets. ${ }^{1}$

In Fig. 4 we attempt a quantitative comparison between the Fe and Co $W_{L M D A D}$ data and the magnetic moment values derived from neutron measurements by Collins and Forsyth. The Fe $W_{L M D A D}$ has been referred to the iron magnetic moment of $\mathrm{Fe}_{0.3} \mathrm{Co}_{0.7}$ which is saturated to the value of $3 \mu_{B}$. The scaling of Fe $W_{L M D A D}$ data for the other stoichiometries follows the trend of scaling of the iron magnetic moment, but with quantitative differences to be discussed below. A similar analysis is shown for Co data in the lower panel of Fig. 4. These results show that the LMDAD measure is indeed sensitive to a systematic variation of the magnetic moments for the elements forming a ferromagnetic alloy while being highly sensitive to the surface, and it can be compared to other techniques.

The quantitative analysis of surface sensitive experimental data requires us to control more parameters than in bulk sensitive measurements: (a) the uncertain value of the surface sensitivity, and (b) the effects of surface magnetism (e.g., surface enhanced magnetic moments). The observed $15 \%$ variation of $W_{L M D A D}$ of Fe in the alloys with respect to clean bcc $\mathrm{Fe}(100)$ is strikingly different with respect to the $36 \%$ increase of bulk moments as measured by neutrons. If we maintain the hypothesis of linear dependence between
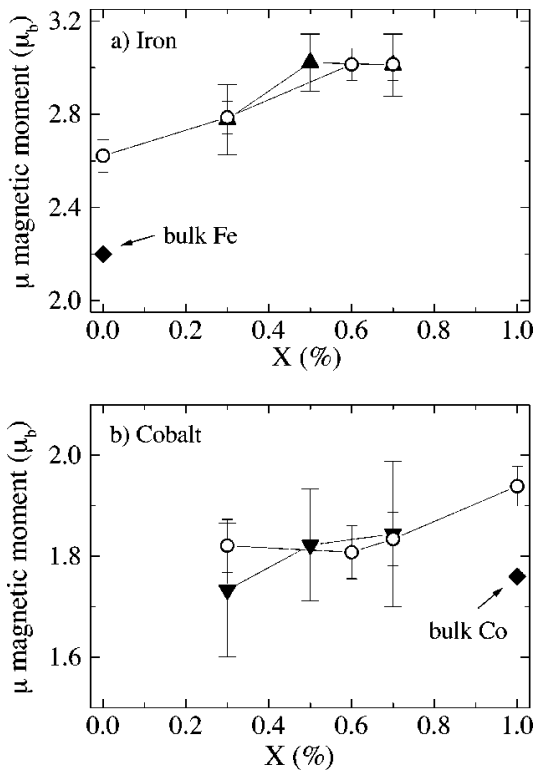

FIG. 4. Neutron data (triangles) ${ }^{23}$ and $W_{L M D A D}$ data (circles) for $\mathrm{Fe}_{1-x} \mathrm{Co}_{x}$ alloys are plotted with the magnetic moment scale of Collins et al. (a) Iron: The Fe $W_{L M D A D}$ data $(\bigcirc)$ are normalized onto the reference data $(\boldsymbol{\Delta})$ for $\mathrm{Fe}_{30} \mathrm{Co}_{70}$. The bulk magnetic moment $\left(2.2 \mu_{B}\right)$ is shown $(\bullet)$. (b) Cobalt: The Co $W_{L M D A D}$ data $(\bigcirc)$ are normalized onto the reference data $(\boldsymbol{\nabla})$ for the $\mathrm{Fe}_{30} \mathrm{Co}_{70}$ composition. The bulk magnetic moment $\left(1.76 \mu_{B}\right)$ is shown $(\diamond)$.

$W_{L M D A D}$ and $\mu_{\mathrm{Fe}}$ the result can be understood semiquantitatively by considering that the surface effect on the magnetic moment of iron at $\mathrm{Fe}(100)$ is very large $\left(\sim 3 \mu_{B}\right.$ at the surface $^{45,46}$ against $2.2 \mu_{B}$ in the bulk, i.e., a surface magnetic moment enhancement of $35 \%$ ). This is due to the fact that the surface layer of $\mathrm{Fe}(100)$ is basically a strong ferromagnet (narrowing of the $d$ band at the surface and filling of the majority spin sub-band). ${ }^{46,47}$ The measured $W_{L M D A D}$ value for the clean $\mathrm{Fe}(100)$ surface is the average of the surface enhanced moment of $\mathrm{Fe}(100)$ and of the subsurface and bulk contributions, it correspond to an average magnetic moment of $\sim 2.6 \mu_{B}$, i.e., higher than the bulk reference moment. The surface enhancement of $\mu_{\mathrm{Fe}}$ for Co-Fe alloys can only be smaller then for clean $\mathrm{Fe}(100)$ : the bulk value of $\mu_{\mathrm{Fe}}$ in the alloy increases towards $3 \mu_{B}$ due to the Co-Fe band structure (filling of the majority- $d$ band), reducing the possible residual surface enhancement. With these considerations in mind the agreement between the Fe $W_{L M D A D}$ and the bulk$\mu_{\mathrm{Fe}}$ results is good, albeit it is impossible to make accurate quantitative conclusions since the very value of the surface enhancement depends on details of atomic and electronic structure. The surface enhancement of $\mu_{\mathrm{Co}}$ in pure Co is of the order of $10 \%{ }^{46}$ The Co $W_{L M D A D}$ data and the bulk $\mu_{\mathrm{Co}}$ data of the Co-Fe alloys show a closer agreement than the Fe data.

Table I also reports the magnitude of the LMDAD asymmetry for the two elements across the composition range. The LMDAD order parameter for $\mathrm{Fe}$ is stable in Fe-rich alloys and increases in Fe-diluted alloys along with the increase of $W_{L M D A D}$. Similarly the $A_{L M D A D}$ of Co increases for diluted $\mathrm{Co}$ alloys but the corresponding value of $W_{L M D A D}$ decreases. The Curie temperature of bulk Co-Fe alloys increases from that of pure $\mathrm{Fe}\left(T_{C}=1050 \mathrm{~K}\right)$ to $\mathrm{CoFe}$ 
$\left(T_{C}=1270 \mathrm{~K}\right.$ ) to about $T_{C}=1400 \mathrm{~K}$ for diluted $\mathrm{Fe}$ in Co. ${ }^{48}$ The bcc lattice parameter of the alloys decreases from $2.87 \AA$ for pure Fe to $2.85 \AA$ for an alloy $\mathrm{Fe}_{0.3} \mathrm{Co}_{0.7} \cdot{ }^{16,19}$ In the case of Co diluted in $\mathrm{Fe}$, we have the $\mathrm{Co}$ atom surrounded by high magnetic-moment $\mathrm{Fe}$ atoms. In the case of Fe diluted in $\mathrm{Co}$, the increase in the Fe magnetic moment and the decrease in the interatomic distances increase the exchange interaction between the $\mathrm{Fe}$ and $\mathrm{Co}$ neighbors. Both effects may explain the increase of $A_{L M D A D}$ for the diluted species.

\section{CONCLUSIONS}

We have grown epitaxially ultrathin layers of bcc Fe-Co alloys on a $\mathrm{Fe}(100)$ substrate by coevaporation in UHV conditions. We have measured the magnetic dichroism LMDAD on the $3 p$ core levels of $\mathrm{Fe}$ and $\mathrm{Co}$ as a function of the alloy composition. The dichroism verifies the ferromagnetic order and the ferromagnetic coupling between the ultrathin alloy film and the iron substrate. We have analyzed the LMDAD splitting $\left(W_{L M D A D}\right)$ as a function of the Co concentration and we have compared the results with the reference data for clean surfaces and for bulk alloys. The changes of $W_{L M D A D}$ for each element are clearly linked to the respective local magnetic moment variations. The "strong ferromagnet" behavior of the clean $\mathrm{Fe}(100)$ surface is converted in the strong ferromagnetic behavior of the alloy as the concentration of the surface alloy changes. The qualitative variation of the Co and Fe moments as a function of composition is similar as in the bulk alloys. This study proves that $W_{L M D A D}$ can be used as a diagnostic for the changes of magnetic moment of the elements contributing to the total magnetization of a ferromagnetic alloy. The strong surface sensitivity of photoemission allows us to study ultrathin epitaxial films on a substrate that can be of the same material of one element composing the alloy. The substrate contribution is negligible for overlayers as thin as ten atomic layers. The analysis of the $W_{L M D A D}$ adds information with respect to the measure of the asymmetry $A_{L M D A D}$ which represents an order parameter of magnetization at the surface. A reduction of $A_{L M D A D}$ due to thermal disorder for example, or to domains, does not imply a reduction of $W_{L M D A D}$ if locally the bonding configuration and consequently the local magnetic moment are well defined. However, the LMDAD measurements can hardly be converted in an absolute magnetometry. This is due both to the incertitude on the effective surface sensitivity of the data, to the existence of surface and subsurface enhancements of the magnetic moments, and to the lack of knowledge of the exact dependence of $W_{L M D A D}$ upon the local magnetic moment.

\section{ACKNOWLEDGMENT}

M.L. acknowledges a INFM grant while working on this project for his Laurea thesis.
${ }^{1}$ F. Sette, in Proceedings of the NATO Advanced Study Institute New Directions in Research with Third-Generation Soft X-Ray Synchrotron Radiation Sources, edited by A. S. Schlachter and F. J. Wuilleumier (Kluwer Academic, Dordrecht, 1994), p. 251; J. Stohr and Y. Wu, ibid., p. 221.

${ }^{2}$ M. Sacchi, C. F. Hague, E. M. Gullikson, and J. H. Underwood, Phys. Rev. B 57, 108 (1998).

${ }^{3}$ M. Sacchi and A. Mirone, Phys. Rev. B 57, 8408 (1998).

${ }^{4}$ L. Baumgarten, C. M. Schneider, H. Petersen, F. Schafers, and J. Kirschner, Phys. Rev. Lett. 65, 492 (1990); H. Ebert, L. Baumgarten, C. M. Schneider, and J. Kirschner, Phys. Rev. B 44, 4406 (1991).

${ }^{5}$ Ch. Roth, F. U. Hillebrecht, H. Rose, and E. Kisker, Phys. Rev. Lett. 70, 3479 (1993); Solid State Commun. 86, 647 (1993).

${ }^{6}$ F. Sirotti and G. Rossi, Phys. Rev. B 49, 15682 (1994).

${ }^{7}$ M. Getzlaff, Ch. Ostertag, G. H. Fecher, N. A. Cherepkov, and G. Schonhense, Phys. Rev. Lett. 73, 3030 (1994).

${ }^{8}$ G. van der Laan, Phys. Rev. Lett. 66, 2527 (1991); G. van der Laan, M. A. Hoyland, M. Surman, C. F. J. Flipse, and B. T. Thole, ibid. 69, 3827 (1993).

${ }^{9}$ M. Altarelli and P. Sainctavit, in Magnetism and Synchrotron Radiation, edited by E. Beaurepaire, B. Carrilere, and J.-P. J. Kappler (Les Editions de Physique, Les Ulis, 1997).

${ }^{10}$ F. Sirotti, G. Panaccione, and G. Rossi, Phys. Rev. B 52, R17 063 (1995).

${ }^{11}$ G. Rossi, F. Sirotti, N. A. Cherepkov, F. Combet-Farnoux, and G. Panaccione, Solid State Commun. 90, 557 (1994).

${ }^{12}$ N. A. Cherepkov, Phys. Rev. B 50, 13813 (1994).

${ }^{13}$ D. Venus, Phys. Rev. B 49, 8821 (1994).

${ }^{14}$ E. Tamura, G. D. Waddill, J. G. Tobin, and P. A. Sterne, Phys. Rev. Lett. 73, 1533 (1994).
${ }^{15}$ G. van der Laan, Phys. Rev. B 51, 240 (1995).

${ }^{16}$ E. Jartych, J. K. Zurawicz, and M. Budzyski, J. Phys.: Condens. Matter 5, 927 (1993).

${ }^{17}$ M. Wojcik, J. P. Jay, P. Panissod, E. Jedryka, J. Dekoster, and G. Langouche, Z. Phys. B 103, 5 (1997).

${ }^{18}$ P. C. Riedi, T. Dumelov, M. Rubinstein, G. A. Prinz, and S. B. Qadri, Phys. Rev. B 36, 4595 (1987).

${ }^{19}$ G. A. Prinz, Phys. Rev. Lett. 54, 1051 (1985).

${ }^{20}$ A. Y. Liu and D. J. Singh, J. Appl. Phys. 73, 6189 (1993).

${ }^{21}$ P. Weiss and R. Forrer, Ann. Phys. (Paris) 12, 279 (1929).

${ }^{22}$ D. I. Bardos, J. Appl. Phys. 40, 1371 (1969).

${ }^{23}$ M. F. Collins and J. B. Forsyth, Philos. Mag. 8, 401 (1963).

${ }^{24}$ S. Pizzini, A. Fontaine, E. Dartyge, C. Giorgetti, F. Baudelet, J. P. Kappler, P. Boher, and F. Giron, Phys. Rev. B 50, 3779 (1994).

${ }^{25}$ P. Söderlind, O. Eriksson, B. Johansson, R. C Albers, and A. M. Boring, Phys. Rev. B 45, 12911 (1992).

${ }^{26}$ R. H. Victora and L. M. Faicov, Phys. Rev. B 30, 259 (1984).

${ }^{27}$ N. Hamada, J. Phys. Soc. Jpn. 46, 1759 (1979).

${ }^{28}$ I. Turek, J. Kudrnovský, V. Drchal, and P. Weinberger, Phys. Rev. B 49, 3352 (1994).

${ }^{29}$ R. Richter and H. Eschrig, J. Phys. F 18, 1813 (1988).

${ }^{30}$ A. Y. Liu and D. J. Singh, Phys. Rev. B 46, 11145 (1992).

${ }^{31}$ G. Rossi, F. Sirotti, and G. Panaccione, in Core Level Spectroscopies for Magnetic Phenomena: Theory and Experiment, Vol. 345 of NATO Advanced Study Institute, Series B: Physics, edited by P. Bagus, G. Pacchioni, and F. Parmigiani (Plenum, New York, 1995).

${ }^{32}$ G. Panaccione, Ph.D. thesis, Univ. Paris VI and LURE, Orsay, France, 1995; G. Panaccione, F. Sirotti, and G. Rossi, J. Electron Spectrosc. Relat. Phenom. 76, 189 (1995). 
${ }^{33}$ G. Rossi, G. Panaccione, F. Sirotti, S. Lizzit, A. Baraldi, and G. Paolucci, Phys. Rev. B 55, 11488 (1997).

${ }^{34}$ G. van der Laan and B. T. Thole, Solid State Commun. 92, 427 (1994).

${ }^{35}$ B. T. Thole and G. van der Laan, Phys. Rev. B 50, 11474 (1994).

${ }^{36}$ G. Panaccione, F. Sirotti, E. Narducci, and G. Rossi, Phys. Rev. B 55, 389 (1997).

${ }^{37}$ G. Panaccione, P. Torelli, G. Rossi, G. van der Laan, M. Sacchi, and F. Sirotti, Phys. Rev. B 58, R5916 (1998).

${ }^{38}$ F. U. Hillebrecht, H. B. Rose, T. Kinoshita, Y. U. Idzerda, G. van der Laan, R. Denecke, and L. Ley, Phys. Rev. Lett. 75, 2883 (1995).

${ }^{39}$ R. Schellenberg, E. Kisker, A. Fanelsa, H. U. Hillebrecht, J. G. Menchero, A. P. Kaduwela, C. S. Fadley, and M. A. Van Hove, Phys. Rev. B 57, 14310 (1998).

${ }^{40}$ R. Schellenberg, E. Kisker, M. Faust, A. Fanelsa, and F. U. Hillebrecht, Phys. Rev. B 58, 81 (1998).
${ }^{41}$ F. Sirotti, G. Panaccione, and G. Rossi, Phys. Rev. B 52, R17 063 (1995).

${ }^{42}$ W. C. Ellis and E. S. Greiner, Trans. Am. Soc. Met. 29, 415 (1941).

${ }^{43}$ F. Sirotti, M. DeSantis, and G. Rossi, Phys. Rev. B 48, 8299 (1993).

${ }^{44}$ Y. U. Idzerda, C. J. Gutierrez, L. H. Tjeng, H.-J. Lin, G. Meigs, and C. T. Chen, J. Magn. Magn. Mater. 127, 109 (1993).

${ }^{45}$ C. L. Fu, A. J. Freeman, and T. Oguchi, Phys. Rev. Lett. 54, 2700 (1985).

${ }^{46}$ O. Eriksson, G. W. Fernando, R. C. Albers, and A. M. Boring, Solid State Commun. 78, 801 (1991).

${ }^{47}$ S. R. Chubb and W. E. Pickett, Phys. Rev. B 38, 10227 (1988).

${ }^{48}$ Magnetische Eigenshaften I, edited by H. A. Alperin et al., Landolt-Börnstein, Band 2, Teil 9 (Springer-Verlag, Berlin, 1962), p. I-53. 